\title{
Retraction Note: Three-year Experience with Alendronate Treatment in Postmenopausal Osteoporotic Japanese Women with or without Renal Dysfunction: A Retrospective Study
}

\author{
Jun Iwamoto ${ }^{1} \cdot$ Yoshihiro Sato $^{2} \cdot$ Mitsuyoshi Uzawa $^{3} \cdot$ Tsuyoshi Takeda $^{1} \cdot$ Hideo Matsumoto ${ }^{1}$
}

Published online: 16 August 2019

(c) Springer Nature Switzerland AG 2019

\section{Retraction Note: Drugs Aging 2012; 29(2):133-142 https://doi.org/10.2165/11598440- 000000000-00000}

The Editor has retracted this article [Iwamoto J, Sato Y, Uzawa M, Takeda T, Matsumoto H. Three-year Experience with Alendronate Treatment in Postmenopausal Osteoporotic Japanese Women with or without Renal Dysfunction: A Retrospective Study. Drugs Aging 2012; 29(2): 133-142.]. After publication, serious concerns were raised with respect to the reporting of the study design, methodology, data and authorship, and the Editor no longer has confidence in this article. Hideo Matsumoto and Tsuyoshi Takeda agree with this retraction. Jun Iwamoto and Mitsuyoshi Uzawa have not responded to correspondence about this retraction. Yoshihiro Sato is deceased.

The original article can be found online at https://doi. org/10.2165/11598440-000000000-00000.

Jun Iwamoto

jiwamoto@a8.keio.jp

1 Institute for Integrated Sports Medicine, Keio University School of Medicine, 35 Shinanomachi, Shinjuku-ku, Tokyo 160-8582, Japan

2 Department of Neurology, Mitate Hospital, Fukuoka, Japan

3 Department of Orthopaedic Surgery, Keiyu Orthopaedic Hospital, Gunma, Japan 